\title{
Polyphenols weaken pea protein gel by formation of large aggregates with diminished non-covalent interactions
}

Da Chen,$^{\dagger}$ Xiao Zhu, ${ }^{\zeta}$ Jan Ilavsky, ${ }^{\ddagger}$ Tanya Whitmer,${ }^{\delta}$ Emmanuel Hatzakis,${ }^{\dagger}$ Owen G. Jones, ${ }^{\tau}$ Osvaldo H. Campanella ${ }^{\dagger, *}$

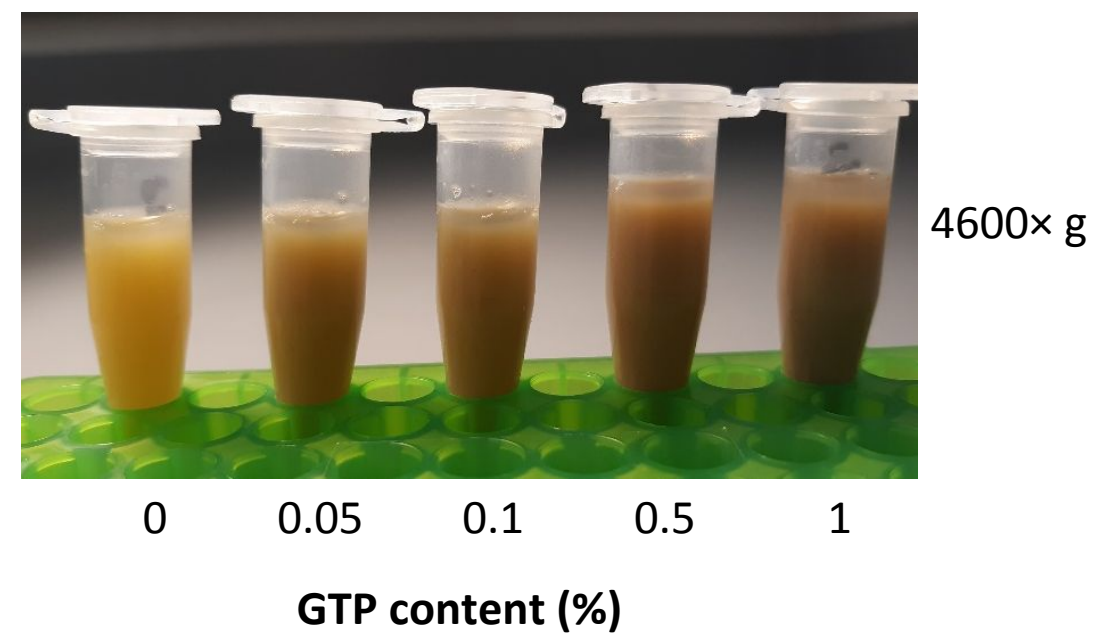

Figure S1. Pea protein solutions of various green tea polyphenols centrifuged at $4600 \mathrm{~g}$ force. Only slight serum layer was observed indicating high stability of the protein solutions. 
a

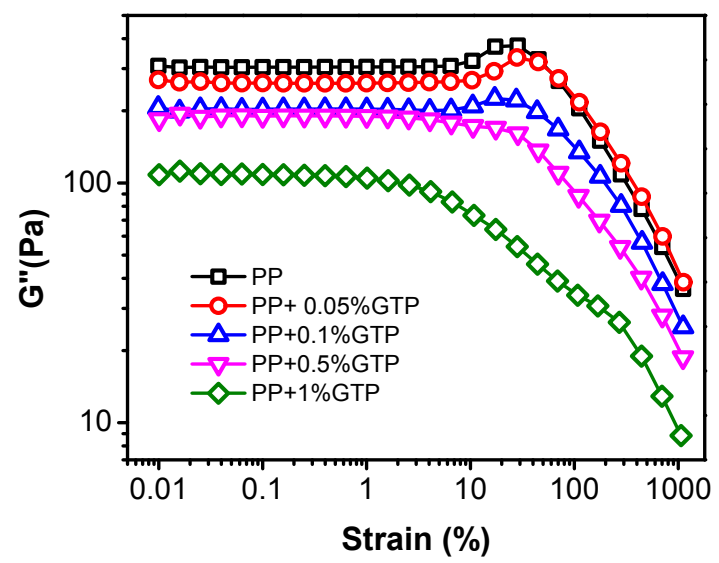

b

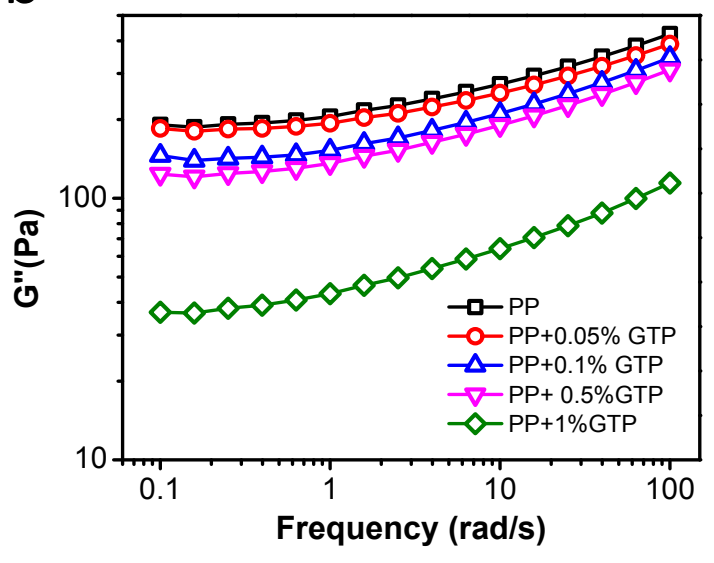

Figure S2. Loss moduli of pea protein gels containing various amount of GTP. (a) Amplitude sweep. (b) Frequency sweep. 


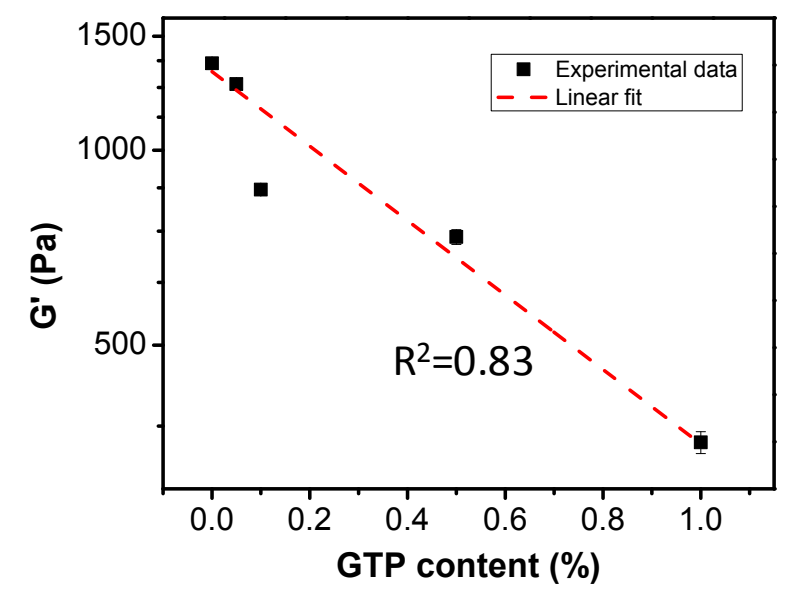

Figure S3. Correlation between the G' of the linear viscoelastic region and the content of GTP. The correlation was fitted with a linear curve with a $\mathrm{R}^{2}$ of 0.83 .

\section{Further explanation of the viscosity and complex viscosity}

The complex viscosity $\left(\eta^{*}\right)$ is a frequency-dependent viscoelastic parameter measured by a Small Amplitude Oscillatory Shear (SAOS) test. Under the condition of small amplitude for either the stress or the strain (strain used in this article), it can be assumed that the structure of the tested material does not change, in other others words the SAOS test can be considered a non-destructive test. One way to assess that the material is not disturbed during testing is using the strain sweep test (please refer to Figure 2a). In that test and the resulting figure, the elastic component of the gel $\left(\mathrm{G}^{\prime}\right)$ does not change with the deformation (strain) until a strain where the elastic component of the gel starts to decrease is achieved. In rheological terms that critical strain is an indication that for higher strains (or stresses if stress is used as the control variable) the sample is disturbed. Another use of the strain sweep test is to evaluate the resistance of gels or viscoelastic materials to be fractured/disturbed when strains (or stresses) are applied. In other words, the test can quantify the ability of the material to resist the application of shear forces (either using strain or stress). That critical strain defines the lineal and not lineal viscoelastic regions of the sample, which is essential because all the other viscoelastic tests need to be conducted and are only valid when the strain used is within the linear region. There are options to analyse the data in the non-lineal viscoelastic region, but the analysis is cumbersome and results so far have not shown good reproducibility for complex systems as the one we are studying.

Under the lineal viscoelastic region (i.e., undisturbed sample) there are other tests commonly used. In the test illustrated in Figure 2c, the storage or elastic moduli $\left(\mathrm{G}^{\prime}\right)$ of the samples are measured as a function of frequency at a fixed strain. Any strain within the linear viscoelastic region, is valid to estimate the storage modulus $G^{\prime}$ and other viscoelastic properties such as the phase angle (see Figure 2b) and the loss modulus G" (not shown in the manuscript). The loss modulus G" is the viscous (or liquid) characteristic of a viscoelastic material, which can exhibit 
both elastic and viscous behaviour. Since in our work all the tested materials were gels, they exhibit a more elastic behaviour and that is the main reason why for these materials $G$ ' $>>G$ '; $G$ " is presented as supplementary information in Figure S2. In summary, in a SAOS test, i.e., done within the lineal viscoelastic region, three main parameters are determined, the phase angle $\delta$ (Fig. 2b), the storage modulus G' (Fig. 2c) and the loss modulus G' (Fig. S2). Frequency sweep tests (Figs. $2 b$ and $2 c$ ) are very useful to characterize the nature of gels. Practically, a purely elastic material will have only an elastic modulus G', and the loss modulus G' is zero. Furthermore, it has been demonstrated that for a purely elastic material the elastic modulus is independent of the frequency, i.e., the slope of the $G$ ' versus frequency is zero for a purely elastic material. Slopes with values different than zero as indicated in Fig. $2 \mathrm{c}$ is indicating that the studied gels have also a viscous (liquid) component. Furthermore, the change in the values of the slope in Fig 2c are indicating that gels containing higher amount of polyphenols have a more liquid behaviour. The phase angle $\delta$ (Fig. 2b) is also an essential measurement in the SAOS test, in fact is used to estimate the viscoelastic moduli G' and G'. It measures the phase angle between the sinusoidal input (strain in our study) and the sinusoidal output (stress in our study). It has been demonstrated that for a purely elastic material $\delta=0$ (i.e., strain and stress are in phase) and for a purely liquid material $\delta=\pi / 2$ radians or 90 degree. Fig. $2 \mathrm{~b}$ shows the viscoelastic nature of the gels with $0<\delta<90^{\circ}$. Also, it indicates the different behaviours of the gels with different amounts of polyphenols. From these three fundamental parameters other parameters have been derived using different analyses and approaches but essentially the equations to calculate these derived parameters are the same regardless of the analysis.

Two commonly used derived parameters from these three parameters are the dynamic viscosity, $\eta^{\prime}=G^{\prime \prime} / w$, where $w$ is frequency and $\eta^{\prime \prime}$ which is called as the "in phase complex dynamic viscosity" and defined as $\eta^{\prime \prime}=G^{\prime} / w$. The latter is a parameter associated to the elasticity the material (the in-phase characteristic of the gel) which to keep consistency it has been also called viscosity. We agree with the reviewer that sometimes this definition may create confusion. However, it is the terminology used for years in the Rheology area (e.g., see Han, Zhao and Wang 2016, Rheol Acta, 55: 257-266; Li et al. 1997, Polym. Eng. Sci.1997, 37:18-23 among books and hundreds of articles) and trying to change this may induce to further confusion. One of the mathematical approaches used to combine these two viscoelastic parameters is define a "complex viscosity" $\eta^{*}=\eta^{\prime}-i \eta$ " which is a complex number where the real part is associated to the dynamic viscosity, in fact associated to the viscous part, and the imaginary part is associated with the "in phase viscosity" that is associated to the elastic component of the viscoelastic sample. The use of a complex number in the analysis is not arbitrary. In fact, it is originated from the analysis of the physics of waves. In other words, the SAOS test is considered that a shear wave is applied to the sample to characterize its viscoelastic properties. Given the difficult of plotting complex numbers, but still with the idea of using a parameter combining both the viscous and elastic nature of the sample, people in the area are using the modulus of the complex viscosity, which is plotted in Figure $2 \mathrm{~d}$, also called complex viscosity. To clarify this concept highlighted by the reviewer we have changed complex viscosity in the manuscript by the magnitude of the complex viscosity (L342, L 343-344, L355, L364) defined as: 


$$
\left|\eta^{*}\right|=\sqrt{\eta^{\prime 2}+\eta^{\prime \prime 2}}=\sqrt{\frac{G^{\prime 2}}{w^{2}}+\frac{G^{\prime \prime 2}}{w^{2}}}=\frac{\sqrt{G^{\prime 2}+G^{\prime 2}}}{w}
$$

In other words, that magnitude of the complex viscosity is derived from the fundamental $G^{\prime}$ and $G^{\prime \prime}$ moduli. As explained above, these are determined under conditions of small shear forces (either using strain or stress), i.e., under conditions under which the structure of sample is not disturbed. The utility of the test is that the information obtained provides a description of the sample structure. The application of sinusoidal mechanical wave in the SAOS test, implies that the experiment is time dependent (oscillatory), an essential condition to be able to characterize viscoelastic materials.

Conversely, the test to determine the viscosity $\eta$ (also known as shear viscosity) reported in Figure 2e uses different conditions. One the main differences is that shear applied (shear rate) is very large and the structure of the sample is completely disturbed during the test. Rather than using frequency like in the SAOS test, shear rate is used as the input to study the response of the material to shear forces. This viscosity measures the internal resistance (friction) to the shear rate (deformation). Although the shear rate is varied to change the structure of the sample with shear, the value of the viscosity at a particular shear rate can be considered as representative of the structure of the sample for that shear rate (deformation). In other words, it is a steady state value independent of time but depending on the applied shear rate. There are fluids whose viscosity are dependent on time, but that behaviour was not observed in our samples. The behaviour observed in Fig. 2e is called shear thinning because the viscosity decreases with increases of shear rates. Obviously, this was occurring because the structure of the sample was disturbed. The other main difference between the tests to obtain $\eta^{*}$ and $\eta$ is that the first is an unsteady test (oscillatory) whereas the second is a steady state test.

It has been demonstrated that when $w \rightarrow 0$ then $\eta^{\prime} \approx \eta$, or that $G^{\prime \prime}>>G^{\prime}$, or $\eta^{\prime}>\eta^{\prime \prime}$. Considering the definition of complex viscosity given above $\eta^{*} \approx \eta$. This normally applies to the systems without presence of structure/interactions that can be potentially destructed at large deformations. However, for pea protein gels, due to protein aggregation, $\eta^{\prime \prime}$ contribution should not be ignored, thus $\eta^{*}>\eta$ at the same frequency or shear rate. Deviation of $\eta^{*}$ from $\eta$ serves as direct evidence of formation of gel network or presence of long-range interactions between proteins (Li, Zhao and Chen 2006, J. Dispers. Sci. Technol. 2005, 415-419).

Another reason why we intend to compare the two viscosities is to use that comparison, known as the Cox-Mertz rule, to assess if the interactions are due to crosslinking or non-covalent bonds. That was explained in the original manuscript and the presence of non-covalent was confirmed rheologically and using other techniques. The measurement of the viscosities is also helping to understand the effect of the GTP in the gels. When adding GTP into pea protein gels, the difference between $\eta^{*}$ and $\eta$ decreases, indicating the viscosity contribution from the elastic component $\left(\eta^{\prime \prime}\right)$ in the pea protein gel became less important. This implies that GTP disrupts or reduces the formation of a network structure or long-range non-covalent interactions. 
a

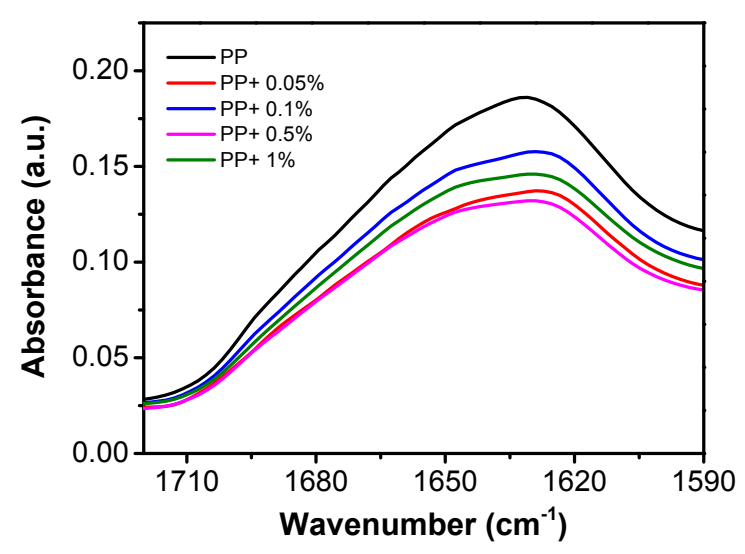

b

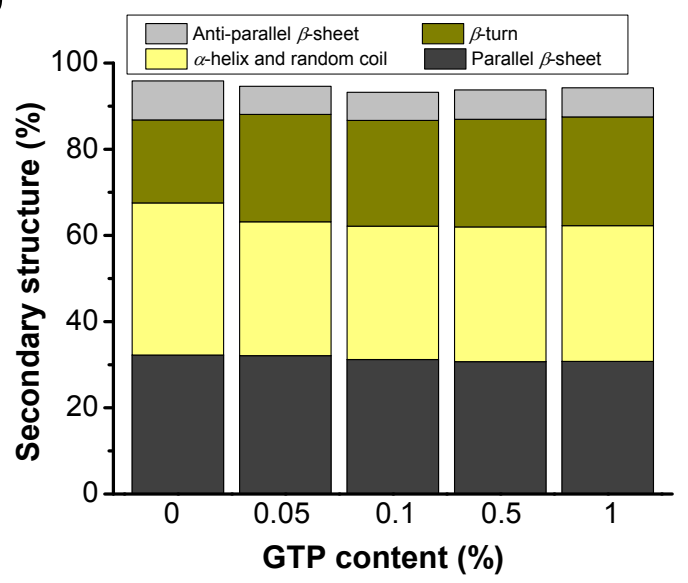

Figure S4. FTIR analysis of secondary structure of proteins in gels containing different amount of GTP. (a) Regions of 1590-1710 $\mathrm{cm}^{-1}$ of protein band. (b) Proportions of different types of secondary structures in the gels.

\section{Attenuated Total Reflection-Fourier Transform Infrared Spectroscopy (ATR-FTIR)}

PPI gels with varied contents of polyphenols were freeze dried. They were then transferred into an ATR accessory of a 4500 Series Portable FTIR (Agilent, Santa Clara, CA). Spectra were recorded over the range $650-4000 \mathrm{~cm}^{-1}$ with 64 scans at a resolution of $4 \mathrm{~cm}^{-1}$. To minimize the interference from polyphenols, their spectra were also recorded and then subtracted from those of PP/GTP gels by multiplying a scaling factor until same signal intensity reaches at 1533 and $1617 \mathrm{~cm}^{-1}$. Regions from 1590 to $1700 \mathrm{~cm}^{-1}$ (amide I) of subtracted spectra were deconvoluted from their secondary derivatives using Peakfit software (Systat Software, Inc.). Peaks centre around $1625,1645,1664,1680 \mathrm{~cm}^{-1}$, representing parallel $\beta$-sheet, $\alpha$-helix and random coil, $\beta$-turn and anti-parallel $\beta$-sheet respectively, were used to estimate the proportion 
of different secondary structures based on ratio of area of individual peak to that of total region. Duplicates were run for each sample and the average results was shown.
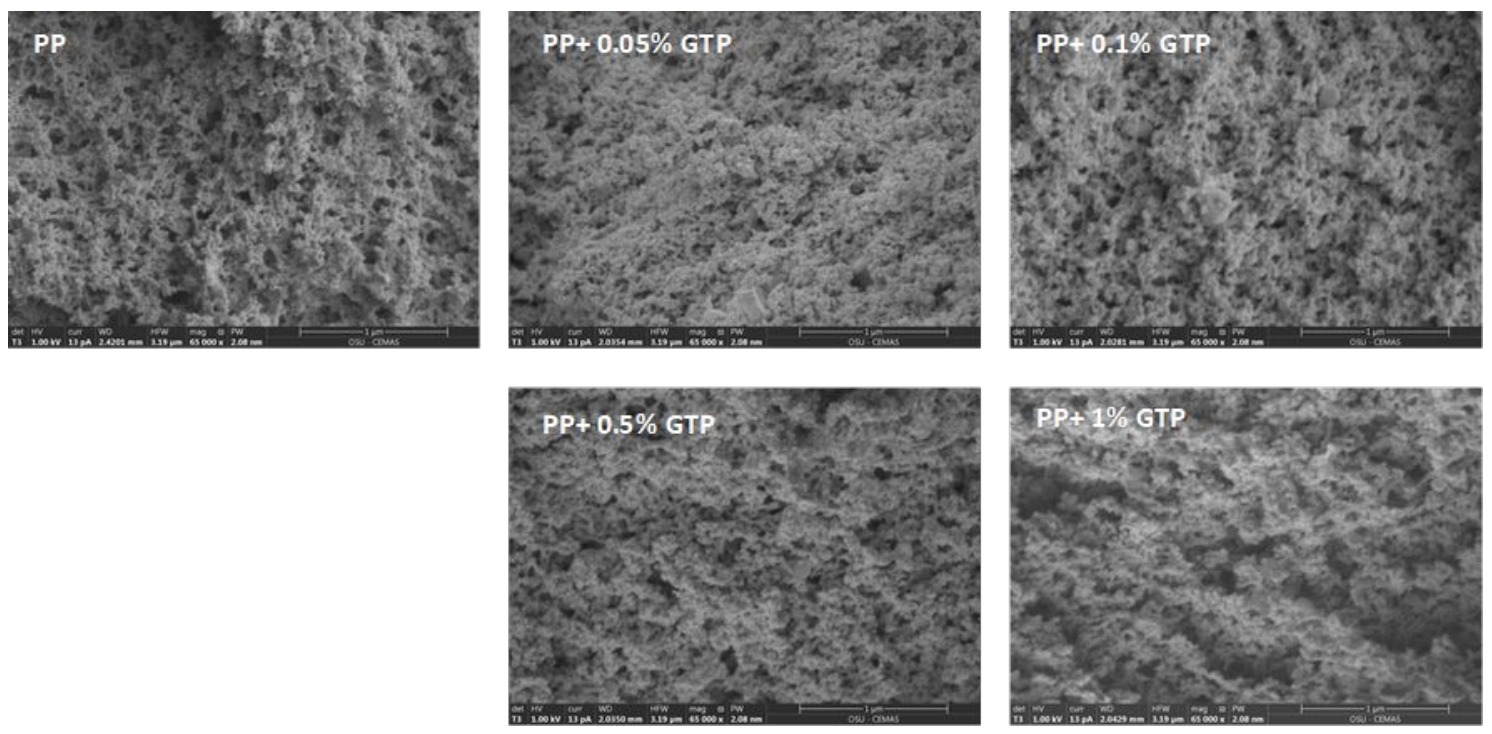

Figure S5. SEM micrographs of pea protein gels without fixation. 


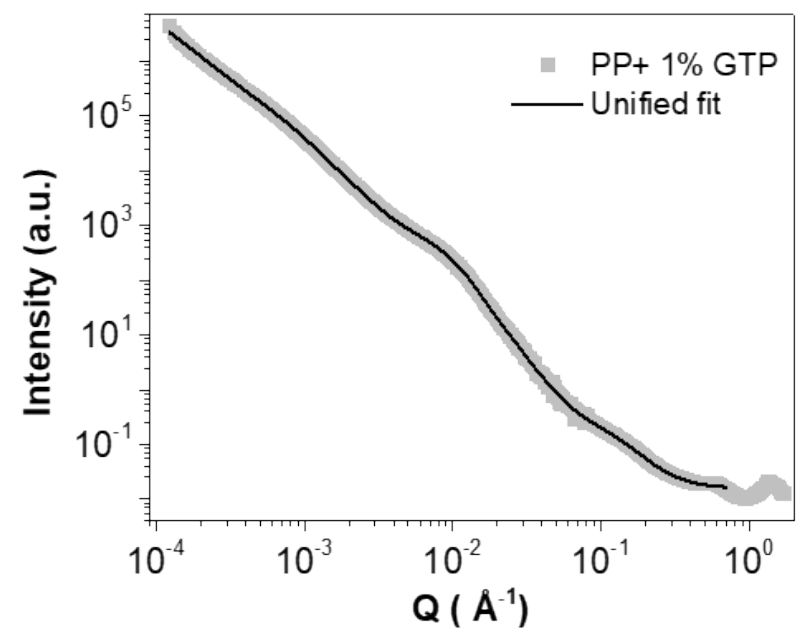

Figure S6. A unified fit of scattering curve of pea protein gel containing 1\%GTP. 


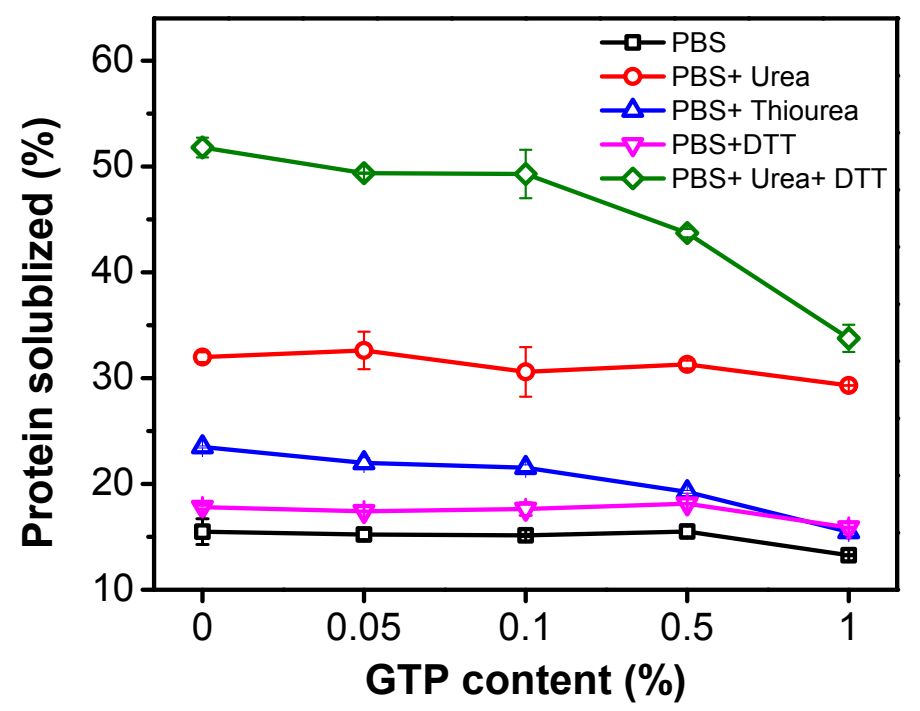

Figure S7. Solubilized protein from pea protein gels extracted with different reagents. 

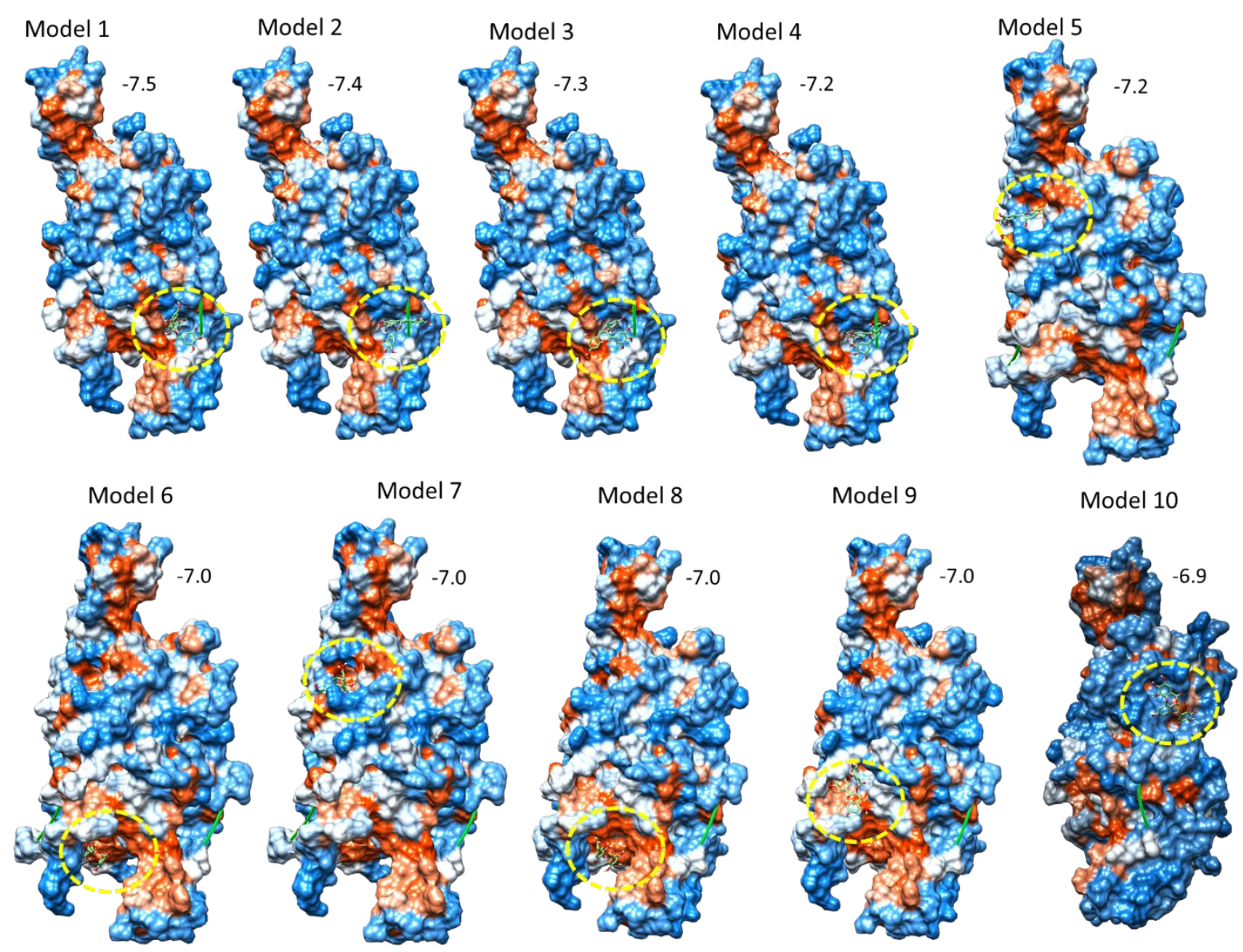

Figure S8. The top ten best binding modes of 11S legumin and EGCG. Of which, models 1-4; 5 and 7; 9; and 6 and 8 bound to the cavities at the region 1,2,3 and 4 respectively as shown in Figure 8. For different models at the same region, EGCG rotates or its position shifts slightly. The locations of the EGCG has been highlighted with dashed circles. The number on the top-right of the protein model denotes the free binding energy with a unit of $\mathrm{kcal} / \mathrm{mol}$. 


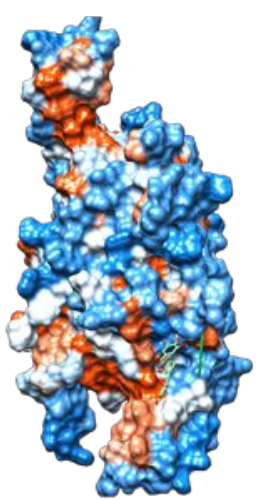

Model 1

Binding energy -7.5

(kcal/mol)

Hydrogen Gln147 HE22-O9

bonds Gln $153 \mathrm{HE} 21-08$

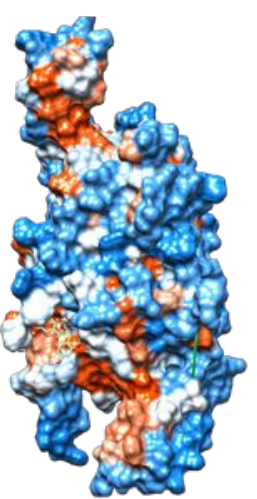

Model 2

$-7.4$

Thr207 HG1-O5

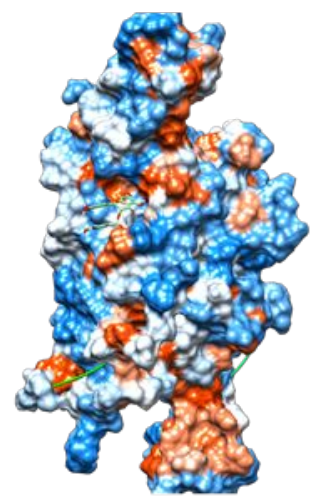

Model 3

$-7.0$

Glu252 OE1-H8

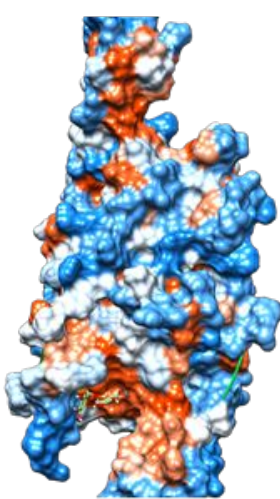

Model 4

$-6.9$

Gln88 H-O7

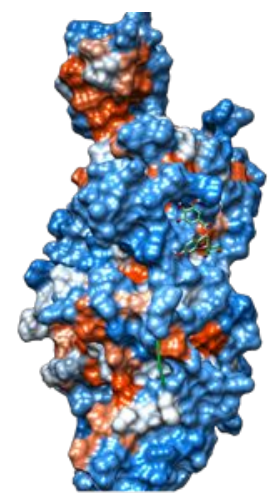

Model 5

$-6.8$

Glu13 H-O4

Glu13 O-H13

Figure S9. Docking results of ECG and 11S legumin. The top five binding modes are shown. Italic fonts denote the atoms are from ECG. 